\title{
Serum Fetuin-A levels are increased and associated with insulin resistance in women with polycystic ovary syndrome
}

Sha Liu

Affiliated Hospital of Zunyi Medical College

\section{Wenjing Hu}

Chongqing Prevention and treatment Hospital for Occupational Diseases https://orcid.org/0000-00031905-7528

\section{Yirui He}

Chongqing Medical University Second Affiliated Hospital

\section{Ling Li}

Chongqing Medical University

\section{Hua Liu}

University of Mississippi Medical Center

\section{Lin Gao}

Affiliated Hospital of Zunyi Medical College

Gangyi Yang ( $\square$ gangyiyang@163.com )

Xin Liao

Affiliated Hospital of Zunyi Medical College

\section{Research article}

Keywords: Fetuin-A, polycystic ovary syndrome, Insulin resistance

Posted Date: May 9th, 2020

DOI: https://doi.org/10.21203/rs.2.24005/v4

License: (c) (i) This work is licensed under a Creative Commons Attribution 4.0 International License. Read Full License

Version of Record: A version of this preprint was published at BMC Endocrine Disorders on May 19th, 2020. See the published version at https://doi.org/10.1186/s12902-020-0538-1. 


\section{Abstract}

Background: Insulin resistance (IR) is a common characteristic of women with polycystic ovary syndrome (PCOS). It has been reported that circulating Fetuin-A levels were associated with IR and type 2 diabetes mellitus (T2DM). However, previous reports were inconsistent.

Methods: 207 subjects were screened for PCOS according to the diagnostic guideline of the Rotterdam consensus criterion. Serum Fetuin-A levels were measured using an ELISA kit. An independent $t$-test or Nonparametric test was used to detect differences between PCOS and control groups. Spearman's correlation analysis was used to examine the association of the serum Fetuin-A with other parameters.

Results: Our findings showed that circulating Fetuin-A concentration ranged from 196.6 to $418.2 \mu \mathrm{g} / \mathrm{L}$ for most women without PCOS (95\%). Women with PCOS had higher circulating Fetuin-A levels than healthy women $(437.9 \pm 119.3 v s .313 .8 \pm 60.5 \mu \mathrm{g} / \mathrm{L} ; p<0.01)$. Serum Fetuin-A was positively correlated with BMI, WHR, TG, TC, LDL-C, HOMA-IR, LH, T, and DHEA-S. Multivariate regression analysis showed that WHR, TG, HOMA-IR, and DHEA-S were independent predictors of the levels of circulating Fetuin-A. Binary logistic regression revealed that serum Fetuin-A was associated with the occurrence of PCOS. In addition, our ROC curve analysis found that the cutoff values for Fetuin-A to predict PCOS and IR were 366.3 and $412.6 \mu \mathrm{g} / \mathrm{L}$.

Conclusion: Blood Fetuin-A may be a useful biomarker for screening women for PCOS and IR.

\section{Background}

Polycystic ovary syndrome (PCOS) is one of the most common endocrine, metabolic diseases in adolescent women. It has three main characteristics: oligo or amenorrhea (OA), hyperandrogenism (HA), and/or clinical manifestations of HA, polycystic ovary (PCO), and most cases are accompanied by obesity and other metabolic disorders. In addition to symptoms caused by hyperandrogenism and reproductive disorders, increasing evidence supports the central role of insulin resistance (IR) and compensatory hyperinsulinemia in the pathogenesis of the PCOS [1]. Furthermore, women with PCOS have an increased risk of developing other metabolic diseases, such as obesity, dyslipidemia, chronic inflammation, metabolic syndrome (MetS), type 2 diabetes mellitus (T2DM), atherosclerosis, and cardiovascular disease (CVD) [2-3]. Overall, IR affects up to $70 \%$ of women with PCOS. Despite extensive research, the mechanisms underlying IR in PCOS patients are not entirely understood [4].

The pathogenesis of PCOS is complex, and its etiology remains unclear. From the definition of PCOS to its phenotype, heterogeneity is an inherent feature of PCOS, and its formation also has heterogeneity. In different phenotypes of PCOS, the relative contribution of excessive androgen and other factors, such as obesity and IR, to the development of PCOS has also been manifested in different ways [5]. Although in the past few decades, many studies have explored the mechanism of metabolic disorders and IR in PCOS patients, the current diagnostic criteria do not include indicators reflecting metabolic disorders and IR [6- 
8]. Therefore, it is essential to look for circulating biomarkers that reflect metabolic disorders and IR in PCOS patients.

Alpha-2-Heremans-Schmid glycoprotein (Fetuin-A) is a $64 \mathrm{kDa}$ glycoprotein and previously considered to be a hepatokine [9]. However, recently, some studies have found that adipose tissue can also express and secrete Fetuin-A [10-11]. Therefore, Fetuin-A is defined as both a hepatokine and an adipokine. Previous studies have shown that Fetuin-A is related to glucose and lipid metabolism and IR, including 1 ) inhibition of insulin action through inhibition of the auto-phosphorylation of insulin receptor tyrosine kinase and glucose transporter 4 (GLUT4); 2) combination with saturated fatty acids may cause Fetuin-A to stimulate chronic inflammation through the Toll-like receptor 4 (TLR4), leading to IR [12-14];3) the mRNA and protein of Fetuin-A are increased in ob/ob mice [14]. 4) impairing of adipocyte function leads to IR [15]; 5) increased Fetuin-A expression is associated with endoplasmic reticulum (ER) stress leading to the development of IR [16]. In human studies, it has been found that polymorphisms in Fetuin-A were related

to T2DM [17] and circulating Fetuin-A levels are elevated or decreased, or unchanged in obese patients, T2DM, non- alcoholic fatty liver disease (NAFLD), MetS, PCOS and CVD, and are either associated with or not associated with impaired glucose tolerance and IR [18-24]. These inconsistent findings on the relationship between Fetuin-A and IR, as well as metabolic diseases, necessitate further investigation.

In this study, we selected newly diagnosed women with PCOS as the subjects of research and evaluated the relationship between circulating Fetuin-A and IR in vivo.

\section{Methods}

\section{Study population}

This study was performed from December 2018 to September 2019. One hundred and twenty-two women with PCOS (PCOS group, 19-37 years old) and eighty-five normal controls ( $\mathrm{N}$ group, 19-32 years old) participated in the current study. The diagnosis of PCOS was based on the Rotterdam consensus criterion, which met two of the following three criteria [25]: 1) hyperandrogenism and/or clinical manifestations of hyperandrogenism; 2) oligo or amenorrhea; 3) ultrasound imaging of polycystic ovary. Other related diseases and disorders were excluded. All PCOS patients were newly diagnosed without lifestyle intervention or any medication. Eighty-five age-matched women were recruited in this study as normal controls. Control subjects had normal menstrual cycles (21-35 days), normal progesterone (P4) level in the luteal phase, and had no acne, hair loss, or hirsutism. Their ovarian morphology was normal by ultrasonography. Exclusion criteria include T2DM, thyroid disease, CVD, liver, and kidney dysfunction. The subjects were recruited from outpatient clinics, daily physical examinations, or advertisements in schools or communities. All subjects signed informed consent before participating in the study. The study was approved by the Human Research Ethics Committee of ZunYi Medical University and performed in accordance with the Helsinki Declaration.

\section{Anthropometric measurement}


Body mass index (BMI) was calculated as weight divided by height squared. Waist circumference and hip circumference were measured by the same observer for calculation of waist-to-hip ratio (WHR). The formula of homeostasis model assessment of IR ( $\left.\mathrm{HOMA}_{-\mathbb{R}}\right)$ was fasting insulin $(\mathrm{Flns}, \mathrm{IU} / \mathrm{ml}) \times$ fasting blood glucose (FBG, mmol/L)/22.5 [26]. According to $\mathrm{HOMA}_{-{ }_{\mid \mathrm{R}} \text {, }}$ individuals were divided into IR (HOMA- ${ }_{-1 \mathrm{R}}$ 2 3.8) and non-IR (HOMA-IR < 3.8) [27]. After 12-14 hours of fasting, blood samples were obtained from all the subjects before breakfast and centrifuged at $4{ }^{\circ} \mathrm{C}$. The serum was stored at $-80^{\circ} \mathrm{C}$ for further analysis.

\section{Measurements of serum Fetuin-A, sex hormone, and biochemical parameters}

Blood glucose, HbA1c, insulin levels, and blood fat, including triglyceride (TG), total cholesterol (TC), lowdensity lipoprotein cholesterol (LDL-C), and high-density lipoprotein cholesterol (HDL-C) were measured as in a previous population [28].The electrochemiluminescence immunoassay was performed for measuring serum follicle-stimulating hormone (FSH), luteinizing hormone ( $\mathrm{LH})$, and testosterone $(\mathrm{T})$ by using COBASE immunoassay analyzers (Roche Diagnostics $\mathrm{GmbH}$ ). An automated analyzer was used for measuring dehydroepiandrostenedione sulfate (DHEA-S) [29].

The serum concentration of Fetuin-A was determined by enzyme-linked immunosorbent assay (ELISA) according to the manufacturer's instructions. Both intra- and inter-assay variations were $10 \%$ and $8 \%$, respectively. The measuring range of this kit was $9.38-600 \mathrm{ng} / \mathrm{ml}$. For the determination of sex hormones, venous blood samples in the healthy controls were collected from day 3 to 5 of the menstrual cycle (early follicular period), and in women with PCOS, blood samples were collected after spontaneous bleeding or amenorrhea for more than three months[30].

\section{Statistical analysis}

Statistical analyses were performed by using SPSS software version 19.0 (SPSS, Chicago, IL). Results were presented as mean \pm SD or median (interquartile range) unless indicated otherwise. The variables of a non-normal distribution were transformed through logarithm before analysis. An independent sample $t$ test or Nonparametric test was performed for comparisons between two groups. One-way ANOVA with post hoc analysis was used to investigate differences in body composition and other indicators between PCOS women and healthy controls. Simple and multiple linear regression analysis was used to study the correlation between fasting Fetuin-A concentrations and other biomarkers. The association of Fetuin-A with PCOS was assessed by multivariate logistic regression analysis. The sensitivity and specificity of Fetuin-A for predicting PCOS were evaluated by a receiver operating characteristic (ROC) curve. Sample size was calculated using the following equations: $N=[Z a / 2 \sigma / \varepsilon \mu] 2(\sigma$, standard; $\mu$, mean; $Z a / 2=1.96, a$ $=0.05, \varepsilon=10 \%) . p<0.05$ was considered significant.

\section{Results}

\section{Serum Fetuin-A concentration in PCOS and healthy women}


Table 1 summarized the demographic, anthropometric, and metabolic parameters and sex hormone levels of all women in the current study. The distribution of Fetuin-A concentrations in healthy women was shown in Figure 1A. We found that circulating Fetuin-A concentration ranged from 196.6 to 418.2 $\mu \mathrm{g} / \mathrm{L}$ for most healthy women (95\%). PCOS patients had higher circulating Fetuin-A levels than healthy women (Figure 1B, Table 1). PCOS patients were divided into obese/overweight (ob/ow) and lean groups by $\mathrm{BMI}<25 \mathrm{~kg} / \mathrm{m}^{2}$ or $\geq 25 \mathrm{~kg} / \mathrm{m}^{2}$, respectively. In all study population, we found that the levels of Fetuin-A in obese/overweight group were significantly higher than those in the lean group (Figure 1C). When healthy women were divided into overweight and lean groups, there was no significant difference in serum Fetuin-A ( $313.5 \pm 60.6$ vs. $317.4 \pm 62.7 \mu \mathrm{g} / \mathrm{L})$, suggesting that there was no significant relationship between serum Fetuin-A level and overweight in healthy women. Furthermore,

Serum Fetuin-A was divided into three concentrations (quantile $1<322.6 \mu \mathrm{g} / \mathrm{L}$; quantile 2

322.6-419.6 $\mu \mathrm{g} / \mathrm{L}$; quantile $3>419.6 \mu \mathrm{g} / \mathrm{L}$ ). The results showed that the incidence of PCOS was significantly higher in the quantile 2 and 3 than in the quantile 1 (95\% $\mathrm{Cl} 1.28-5.14$ in quantile 2 and $95 \%$ Cl 11.8 - 113.5 in quantile 3; Figure 1D).

\section{Serum Fetuin-A level and its association with other parameters in the study population}

Next, we investigated the relationship between the levels of circulating Fetuin-A and various other parameters. Serum Fetuin-A was positively correlated with BMI, WHR, TG, TC, LDL-C, HOMA ${ }_{I R}, L H$, T, and DHEA-S (Table 2). Moreover, as previously reported, we also found a significant correlation between $T$ and HOMA- $_{-\mathrm{R}}(r=0.351, p<0.01)$. We then performed a multiple stepwise regression to determine variables that had independent associations with serum Fetuin-A. The results showed that only WHR, TG, HOMA- ${ }^{-1 R \text {, }}$ and DHEA-S were independent predictors of the levels of circulating Fetuin-A (Table 2). The multiple regression equation was $Y_{\operatorname{Ig} 10 \text { (Fetuin-A) }}=2.11+0.012 \mathrm{X}_{\text {HOMA-IR }}+0.401 \mathrm{X}_{\mathrm{WHR}}+0.017 \mathrm{X}_{\mathrm{TG}}+0.009 \mathrm{X}_{\text {DHEA-S }}$.

\section{ROC curve analysis}

To explore the prediction of PCOS and IR by blood Fetuin-A, we performed a ROC curve analysis. The results showed that the area under the ROC curves for PCOS (AUC $C_{P C O S}$ ) was 0.82 with a specificity of $83.5 \%$, and sensitivity of $69.7 \%(p<0.01$, Figure $2 A)$, and $A U C_{\mathbb{R}^{R}}$ was 0.80 with a specificity of $81 \%$, and a sensitivity of $72.3 \%$. The best cut-off values for Fetuin-A to detect PCOS and IR were $366.3 \mu \mathrm{g} / \mathrm{L}$, and 412. $6 \mu \mathrm{g} / \mathrm{L}$, respectively.

\section{Discussion}

In the past decade, several population-based studies reported the relationship between circulating FetuinA concentrations and PCOS. However, the conclusions of these studies were contradictory. In previous studies, circulating Fetuin-A levels were increased, decreased, or unchanged in PCOS patients compared with healthy women [22, 31-35]. In the current investigation, we found that circulating Fetuin-A concentrations were markedly elevated in women with PCOS compared with healthy women. Our results 
were consistent with those of Enli et al. [33 -35] but contrary to those of Díaz et al. [22]. The crosssectional nature of the study and population heterogeneity might be related to differences in outcomes. Furthermore, previous studies had small sample sizes, and some had no healthy controls or did not include obese patients with PCOS. In this study, we avoid these shortcomings. In addition, we employed newly diagnosed PCOS women to avoid the effects of medication, lifestyle interventions, and the duration of the disease that were associated with those patients who were under treatment. The reason for the rise in circulating Fetuin-A was unknown. We speculate that the metabolic disorders and hyperandrogenism caused by IR (hyperinsulinemia) may promote the synthesis and release of Fetuin-A in vivo. In addition, increased Fetuin-A might be derived, at least in part, by the status of low-grade inflammation, since inflammatory cytokines, such as CRP, were increased in women with PCOS.

Two previous studies found that Fetuin-A inhibited the insulin receptor tyrosine kinase and Toll-like receptor 4 in liver and muscle cells to suppress insulin signaling and stimulate inflammatory signaling pathways $[13,14]$. However, population-based studies showed that there was no correlation between Fetuin-A and IR in diabetic patients, and there was no correlation between Fetuin- $A$ and the risk of diabetes [36-37]. In the current work, we found that serum Fetuin-A was positively correlated with BMI, WHR, TG, TC, LDL-C, HOMA- ${ }_{-1 R}, L H, T$, and DHEA-S, suggesting that Fetuin-A was associated with hyperinsulinemia and hyperandrogenism. These data supported the results of $\mathrm{Pal}$ et al. and Srivas et al. [13]. Because circulating Fetuin-A levels were associated with both $T$ and IR, and hyperandrogenism and IR were essential characteristics of PCOS, it was of clinical significance to consider Fetuin-A a biomarker for PCOS.

Surprisingly, serum Fetuin-A concentration increased significantly in obese/overweight PCOS women but did not change in normal women with obese/overweight. This result was consistent with a previous study [38]. The reason for this was unknown. We speculate that the main factors affecting circulating Fetuin-A might be hyperinsulinemia and hyperandrogenemia, not adipose mass. In our study cohort, a small number of overweight women might affect the results. In addition, under hyperinsulinemia and hyperandrogenism, elevated circulating Fetuin-A raised the question of whether lowering Fetuin-A concentrations were the key to improving IR and hyperandrogenism. Secondly, because serum Fetuin-A levels were related to hyperandrogenemia, it was important to observe whether circulating Fetuin-A concentration would change with the menstrual cycle due to the change of hormone levels. To address these questions, further study is necessary.

We analyzed the ROC curve to explore the best cut-off point for predicting PCOS with circulating Fetuin-A. Our results showed that cyclic Fetuin-A was a good predictor for PCOS patients. Thus, we consider that the association of Fetuin-A with PCOS may be due to the high incidence of IR in PCOS population.

Our research also has some limitations. Firstly, the study population is young women, so our results may not apply to an elderly population. Secondly, our results are based on a single measurement of Fetuin-A. Without repeated measures at different time points, the introduction of random measurement errors in determining biochemical variables is possible. Finally, the nature of the cross-sectional study makes it 
impossible for our results to explain the causal relationship between increased Fetuin-A levels and the occurrence of IR and PCOS.

\section{Conclusion}

In conclusion, our results suggested that serum Fetuin-A levels were increased in PCOS patients.

Circulating Fetuin-A concentrations were associated with dyslipidemia, IR, and ovarian hyperandrogenism in women with PCOS.

\section{Declarations}

\section{Ethics approval and consent to participate}

Before participating in the study, all subjects signed informed consent.The study was approved by the Human Research Ethics Committee of ZunYi Medical University

(Ethical Review of Scientific Research No. 10, approved on September 10th, 2016) and is consistent with the institutional guidelines. The study was performed in accordance with the Helsinki Declaration.

Consent for publication Not applicable.

\section{Availability of data and materials}

The datasets used and/or analyzed during the current study are available from the corresponding author on reasonable request.

Competing interests No potential conflicts of interest relevant to this article were reported.

Funding This work was supported by research grants from the National Natural Science Foundation of China (81601214,). The funders played no role in the design of this study, the collection, analysis and interpretation of data or preparation of the manuscript.

Author contributions S.L., W.H., L.L. and Y.H. researched data. H.L. reviewed and edited the manuscript. L.G. contributed to the writing of the manuscript and helpful discussion. G.Y. and X.L. directed the project, contributed to discussion, and wrote, reviewed, and edited the manuscript and were the guarantors of this work and, as such, had full access to all the data in the study and takes responsibility for the integrity of the data and the accuracy of the data analysis. all authors have read and approved the manuscript.

\section{Acknowledgments}

We thank all the individuals who participated in this study.

\section{Abbreviations}


PCOS, Polycystic ovary syndrome; IR, Insulin resistance; T2DM, type 2 diabetes mellitus; BMI, body mass index; MetS, metabolic syndrome; WHR, waist-to-hip ratio; TG, triglyceride; TC, total cholesterol; LDL-C, low-density lipoprotein cholesterol; HDL-C, high-density lipoprotein cholesterol; HOMA-IR, homeostasis model assessment of insulin resistance; FBG, Fasting blood glucose; LH, luteinizing hormone; FSH, follicle-stimulating hormone; T, testosterone; DHEA-S, dehydroepiandrostenedione sulfate; SBP, systolic blood pressure; DBP, diastolic blood pressure; FIns, fasting plasma insulin; $\mathrm{Cl}$, confidence interval; OR, odds ratio; $\mathrm{ROC}$, receiver operating characteristic.

\section{References}

1. Donà G, Sabbadin C, Fiore C, Bragadin M, Giorgino FL, Ragazzi E, Clari G, Bordin L, Armanini D. Inositol administration reduces oxidative stress in erythrocytes of patients with polycystic ovary syndrome. Eur J Endocrinol. 2012; 166: 703-710.

2. Kelley Angela S, Smith Yolanda R, Padmanabhan Vasantha. A Narrative Review of Placental Contribution to Adverse Pregnancy Outcomes in Women With Polycystic Ovary Syndrome. J Clin Endocrinol Metab. 2019;104:5299-315.

3. Reis AM, Honorato-Sampaio K. C-type natriuretic peptide: a link between hyperandrogenism and anovulation in a mouse model of polycystic ovary syndrome. J Clin Sci. 2018;132:905-8.

4. Calan M, Yilmaz O, Kume T, Unal Kocabas G, Yesil Senses P, Senses YM, Temur M, Gursoy Calan O. Elevated circulating levels of betatrophin are associated with polycystic ovary syndrome. Endoc 2016;53: 271-279.

5. Underdal MO, Salvesen $\emptyset$, Schmedes A, Andersen MS, Vanky E. Prolactin and breast increase during pregnancy in PCOS: linked to long-term metabolic health? J Eur J Endocrinol. 2019;180:373-80.

6. Zhang L, Fang X, Li L, Liu R, Zhang C, Liu H, Tan M, Yang G. The association between circulating irisin levels and different phenotypes of polycystic ovary syndrome. J Endocrinol Invest. 2018;41:1401-7.

7. Li M, Yang M, Zhou X, Fang X, Hu W, Zhu W, Wang C, Liu D, Li S, Liu H, Yang G, Li L. Elevated circulating levels of irisin and the effect of metformin treatment in women with polycystic ovary syndrome. J Clin Endocrinol Metab. 2015;100:1485-93.

8. Li X, Zhu Q, Wang W, Qi J, He Y, Wang Y, Lu Y, Wu H, Ding Y, Sun Y. Elevated chemerin induces insulin resistance in human granulosa-lutein cells from polycystic ovary syndrome patients. J FASEB J. 2019;33:11303-13.

9. Rotterdam ESHRE/ASRM-Sponsored PCOS consensus workshop group. Revised 2003 consensus on diagnostic criteria and long-term health risks related to polycystic ovary syndrome (PCOS). J Hum Reprod. 2004;19:41-7.

10. Stefan N, Haring H. The role of hepatokines in metabolism. J Nat Rev Endocrinol. 2013;9:144-52.

11. Jialal I, Devaraj S, Bettaieb A, Haj F, Adams-Huet B. Increased adipose tissue secretion of Fetuin-A, lipopolysaccharide-binding protein and high-mobility group box protein 1 in metabolic syndrome. $J$ 
Atherosclerosis. 2015;241:130-7.

12. Perez-Sotelo D, Roca-Rivada A, Larrosa-Garcia M, Castelao C, Baamonde I, Baltar J, Crujeiras AB, Seoane LM, Casanueva FF, Pardo M. Visceral and subcutaneous adipose tissue express and secrete functional alpha2hsglycoprotein (fetuin a) especially in obesity. J Endocrine. 2017;55:435-46.

12. Sujana C, Huth C, Zierer A, Meesters S, Sudduth-Klinger J, Koenig W, Herder C, Peters A, Thorand B. Association of fetuin-Awith incident type 2 diabetes: results from the MONICA/KORA Augsburg study and a systematic meta-analysis. J Eur J Endocrinol. 2018;178:389-98.

13. Pal D , Dasgupta S , Kundu R, et al. Fetuin-A acts as an endogenous ligand of TLR4 to promote lipidinduced insulin resistance. J Nature Medicine. 2012;18:1279-85.

14. Jialal I, Pahwa R. Fetuin-Ais also an adipokine. Lipids Health Dis. 2019; 18: 73.

15. Dasgupta S, Bhattacharya S, Biswas A, Majumdar SS, Mukhopadhyay S, Ray S, Bhattacharya S. NFkappaBmediateslipid-induced fetuin-A expression

in hepatocytes that impairs adipocytefunction effecting insulin resistance. Biochem J. 2010;429:451462.

16. Ou HY, Wu HT, Hung HC, Yang YC, Wu JS, Chang CJ. Endoplasmicreticulumstress induces the expression of fetuin-A to develop insulin resistance. Endocrinology. 2012; 153:2974-2984.

17. Siddiq A, Lepretre F, Hercberg S, Froguel P, Gibson F. A synonymous coding polymorphism in the alpha2-Heremans-schmid glycoprotein gene is associated with type 2 diabetes in French Caucasians. J Diabetes.2005;54:2477-81.

18. Yoo HJ, Choi KM. Hepatokines as a link between obesity and cardiovascular diseases. J Diabetes Metab J. 2015;39:10-15.

19. El-Deeb TS, Bakkar SM, Eltoony L, Zakhary MM, Kamel AA, Nafee AM, Hetta HF. The adipokine Chemerin and Fetuin-A Serum Levels in Type 2 Diabetes Mellitus: Relation to Obesity and Inflammatory Markers. J Egypt J Immunol.2018;25:191-202.

20. von Loeffelholz C, Horn P,Birkenfeld A L et al. Fetuin A is a Predictor of Liver Fat in Preoperative Patients with Nonalcoholic Fatty Liver Disease. J J Invest Surg. 2016;29:266-74.

21. Kasabri V, Shawakri E, Akour A, Naffa R, Khawaja N, Al-Sarraf I, Bzour J. Cross- sectional correlates of increased IL-18 but reduced fetuin-A and oxytocin with adiposity and blood indices in metabolic syndrome patients with and without prediabetes. Ther Adv Endocrinol Metab.2018;9:329-38.

22. Díaz Marta, Gallego-Escuredo José Miguel, López-Bermejo Abel et al. Low-Dose SpironolactonePioglitazone-Metformin Normalizes Circulating Fetuin-A Concentrations in Adolescent Girls with Polycystic Ovary Syndrome. J Int J Endocrinol. 2018:4192940.

23. Chen X, Zhang Y, Chen Q, Li Q, Li Y, Ling W. Lower plasma fetuin-a levels are associated with a higher mortality risk in patients with coronary artery disease. J Arterioscler Thromb Vasc Biol.2017;37: 2213-2219.

24. Khadir A, Kavalakatt S, Madhu D, Hammad M, Devarajan S, Tuomilehto J, Tiss A. Fetuin-Alevelsare increased in the adipose tissue of diabetic obese humans but not in circulation. J Lipids Health Dis. 
2018; 17: 291.

25. Wild Robert A, Carmina Enrico, Diamanti-Kandarakis Evanthia et al. Assessment of cardiovascular risk and prevention of cardiovascular disease in women with the polycystic ovary syndrome: a consensus statement by the Androgen Excess and Polycystic Ovary Syndrome (AE-PCOS) Society. J J Clin Endocrinol Metab. 2010; 95: 2038-49.

26. Albareda M, Rodríguez-Espinosa J, Murugo M et al. Assessment of insulin sensitivity and beta-cell function from measurements in the fasting state and during an oral glucose tolerance test. J Diabetologia. 2000; 43: 1507-11.

27. Lecube A, Sampol G, Munoz X et al. Insulin resistance is related to impaired lung function in morbidly obese women: a case control study. Diabetes Metab Res Rev 2010; 26: 639-645.

28. Mi Qiao, Li Yanxin, Wang Miao et al. Circulating C1q/TNF-related protein isoform 15 is a marker for the presence of metabolic syndrome. J Diabetes Metab Res Rev. 2019; 35: e3085.

29. Liao X, Liu S, Tang X, Yang D, Liu H, Gao L, Yang G. Circulating CTRP6 Levels are Increased in Overweight or Obese Chinese Individuals and Associated with Insulin Resistance Parameters: A Pilot Study. J Exp Clin Endocrinol Diabetes. 2019;

30. 30. Lai Y, Chen J, Li L, Yin J, He J, Yang M, Jia Y, Liu D, Liu H, Liao Y, Yang G. Circulating Zinc-a2glycoprotein levels and Insulin Resistance in Polycystic Ovary Syndrome. Sci Rep. 2016; 6: 25934.

31. Gulhan I, Bozkaya G, Oztekin D, Uyar I, Kebapcilar AG, Pamuk B. SerumFetuin-A levelsin women with polycystic ovary syndrome. J Arch Gynecol Obstet. 2012;286:1473-6.

32. Enli Y, Fenkci SM, Fenkci V, Oztekin O. SerumFetuin-Alevels, insulin resistance and oxidative stress inwomen with polycystic ovary syndrome. J Gynecol Endocrinol. 2013;29:1036-9.

33. Kozakowski J, Jeske W, Zgliczyński W. Fetuin-Alevelsin lean and obese women with polycystic ovary syndrome. J Endokrynol Pol. 2014;65:371-6.

34. Sak S, Uyanikoglu H, Incebiyik A, Incebiyik H, Hilali NG, Sabuncu T, Sak E. Associations of serum fetuin-A and oxidative stress parameters with polycystic ovary syndrome. J Clin Exp Reprod Med.2018;45:116-21.

35. Abali R, Celik C, Tasdemir N, Guzel S, Alpsoy S, Yuksel A, Celik E. The serum protein a2-HeremansSchmid glycoprotein/fetuin-aconcentration and carotid intima-media thickness in women with polycystic ovary syndrome. J Eur J Obstet Gynecol Reprod Biol.2013;169:45-9.

36. Jensen MK, Bartz TM, Mukamal KJ, Djoussé L, Kizer JR, Tracy RP, Zieman SJ, Rimm EB, Siscovick DS, Shlipak M, Ix JH. Fetuin-A, type 2 diabetes, and risk of cardiovascular diseasein olderadults: the cardiovascularhealth study. J Diabetes Care. 2013; 36: 1222-8.

37. Jensen Majken K, Bartz Traci M, Djoussé Luc et al. Genetically elevated fetuin-A levels, fasting glucose levels, and risk of type 2 diabetes: the cardiovascular health study. J Diabetes Care. 2013; 36: 3121-7. 
38. Kozakowski J, Jeske W, Zgliczyski W. Fetuin-A levels in lean and obese women with polycystic ovary syndrome. J Endokrynologia Polska, 2014; 65: 371-6.

\section{Tables}

Table 1 Main clinical features and circulating Fetuin-A levels in PCOS and healthy women

\begin{tabular}{llll}
\hline Characteristics & $\begin{array}{l}\text { Controls } \\
(\mathrm{n}=85)\end{array}$ & $\begin{array}{l}\text { PCOS } \\
(\mathrm{n}=122)\end{array}$ & $p$-value \\
\hline Age $(\mathrm{yr})$ & $26.0 \pm 3.4$ & $25.3 \pm 3.4$ & 0.114 \\
BMI $\left(\mathrm{kg} / \mathrm{m}^{2}\right)$ & $21.6 \pm 2.9$ & $24.4 \pm 4.4$ & $<0.001$ \\
WHR & $0.81 \pm 0.08$ & $0.88 \pm 0.10$ & $<0.001$ \\
SBP $(\mathrm{mmHg})$ & $113 \pm 8$ & $128 \pm 8$ & $<0.001$ \\
DBP $(\mathrm{mmHg})$ & $75 \pm 7$ & $77 \pm 7$ & 0.045 \\
TG $(\mathrm{mmol} / \mathrm{L})$ & $0.92(066-1.34)$ & $1.37(1.01-2.48)<0.001$ \\
TC $(\mathrm{mmol} / \mathrm{L})$ & $3.94 \pm 0.76$ & $4.64 \pm 0.93$ & $<0.001$ \\
HDL-C $(\mathrm{mmol} / \mathrm{L})$ & $1.23 \pm 0.27$ & $1.18 \pm 0.33$ & 0.278 \\
LDL-C $(\mathrm{mmol} / \mathrm{L})$ & $2.18 \pm 0.58$ & $2.89 \pm 0.58$ & $<0.001$ \\
FBG $(\mathrm{mmol} / \mathrm{L})$ & $4.77 \pm 0.46$ & $5.06 \pm 0.91$ & $<0.01$ \\
FIns $(\mathrm{mU} / \mathrm{L})$ & $7.40(5.55-9.17)$ & $18.00(9.30-27.0 \&) 0.001$ \\
HOMA-IR & $15.0(1.15-2.04)$ & $3.85(1.97-5.83)<0.001$ \\
FSH $(\mathrm{IU} / \mathrm{L})$ & $5.92 \pm 2.00$ & $5.78 \pm 1.36$ & 0.574 \\
LH $(\mathrm{IU} / \mathrm{L})$ & $6.07 \pm 5.14$ & $8.39 \pm 4.94$ & $<0.001$ \\
T $(\mathrm{nmol} / \mathrm{L})$ & $1.01 \pm 0.68$ & $1.44 \pm 0.65$ & $<0.001$ \\
DHEA-S $(\mu \mathrm{\mu mol} / \mathrm{L})$ & $4.50 \pm 1.86$ & $6.58 \pm 2.93$ & $<0.001$ \\
Fetuin-A $(\mu \mathrm{g} / \mathrm{L})$ & $313.8 \pm 60.5$ & $437.9 \pm 119.3$ & $<0.001$ \\
\hline
\end{tabular}

Values are given as mean \pm SD or median (inter quartile range). BMI, Body mass index; WHR, Waist hip ratio; SBP, Systolic blood pressure; DBP, Diastolic blood pressure; FBG, fasting blood glucose; FIns, fasting plasma insulin; TG, Triglyceride; TC, Total cholesterol; HDL-C, Highdensity lipoprotein cholesterol; LDL-C, Low-density lipoprotein cholesterol; HOMA-IR, homeostasis model assessment of insulin resistance; FSH, follicle-stimulating hormone; LH, luteinizing hormone; T, testosterone; DHEA-S, dehydroepiandrosterone sulfate.

Table 2 Correlation analysis of variables associated with circulating Fetuin-A levels in the study populations 


\begin{tabular}{|c|c|c|c|c|}
\hline \multirow[b]{2}{*}{ Variable } & \multicolumn{2}{|c|}{ Simple } & \multicolumn{2}{|c|}{ Multiple } \\
\hline & $r$ & $p$ & $\bar{b}$ & $p$ \\
\hline Age (years) & -0.124 & 0.076 & $\begin{array}{ll}----- \\
\end{array}$ & $\begin{array}{ll}----- \\
\end{array}$ \\
\hline $\operatorname{BMI}\left(\mathrm{kg} / \mathrm{m}^{2}\right)$ & 0.294 & $<0.001$ & ------ & ------ \\
\hline $\mathrm{SBP}(\mathrm{mmHg})$ & 0.076 & 0.279 & ------ & ----- \\
\hline DBP (mmHg) & 0.039 & 0.579 & ----- & ----- \\
\hline WHR & 0.351 & $<0.001$ & 0.401 & $<0.001$ \\
\hline $\mathrm{TG}(\mathrm{mmol} / \mathrm{L})$ & 0.293 & $<0.001$ & 0.017 & $<0.05$ \\
\hline $\mathrm{TC}(\mathrm{mmol} / \mathrm{l})$ & 0.184 & $<0.01$ & ------ & ------ \\
\hline HDL-C $(\mathrm{mmol} / \mathrm{L})$ & -0.106 & 0.128 & ----- & ----- \\
\hline LDL-C (mmol/L) & 0.339 & $<0.001$ & ----- & ----- \\
\hline $\mathrm{HOMA}^{-\mathrm{IR}}$ & 0.511 & $<0.001$ & 0.012 & $<0.001$ \\
\hline FSH (IU/L) & -0.079 & 0.259 & ------ & ------ \\
\hline LH (IU/L) & 0.178 & $<0.05$ & ----- & ----- \\
\hline $\mathrm{T}(\mathrm{nmol} / \mathrm{L})$ & 0.320 & $<0.001$ & ------ & ------ \\
\hline DHEA-S $(\mu \mathrm{mol} / \mathrm{L})$ & 0.330 & $<0.001$ & 0.009 & $<0.01$ \\
\hline
\end{tabular}

Fetuin-A concentration was a non-normal distribution and transformed by logarithm before analysis. Additionally, logistic regression analysis revealed that Fetuin-A was significantly related to PCOS, even after controlling for anthropometric variables, blood lipid and so on (Table 3).

Table 3 Association of circulating Fetuin-A with PCOS in fully adjusted models

\begin{tabular}{|c|c|c|c|}
\hline \multirow[t]{2}{*}{ Model adjustments } & \multicolumn{3}{|c|}{ PCOS } \\
\hline & OR & $95 \% \mathrm{CI}$ & $P$ \\
\hline \multirow[t]{2}{*}{ Age, SBP, DBP } & 1.015 & $1.010-$ & \\
\hline & & 1.020 & 0.000 \\
\hline \multirow[t]{2}{*}{ Age, SBP, DBP, BMI, WHR } & 1.014 & $1.009-$ & \\
\hline & & 1.019 & 0.000 \\
\hline \multirow[t]{2}{*}{ Age, SBP, DBP, BMI, WHR, FBG, FIns } & 1.011 & $1.005-$ & \\
\hline & & 1.017 & 0.000 \\
\hline \multirow[t]{2}{*}{ Age, SBP, DBP, BMI, WHR, FPG, FIns, Lipid profile } & 1.010 & $1.004-$ & \\
\hline & & 1.017 & 0.002 \\
\hline $\begin{array}{l}\text { Age, SBP, DBP, BMI, WHR, FPG, FIns, Lipid profile, Hormone } \\
\text { parameters }\end{array}$ & 1.008 & $\begin{array}{l}1.001- \\
1.016\end{array}$ & 0.036 \\
\hline
\end{tabular}



groups.

\section{Figures}
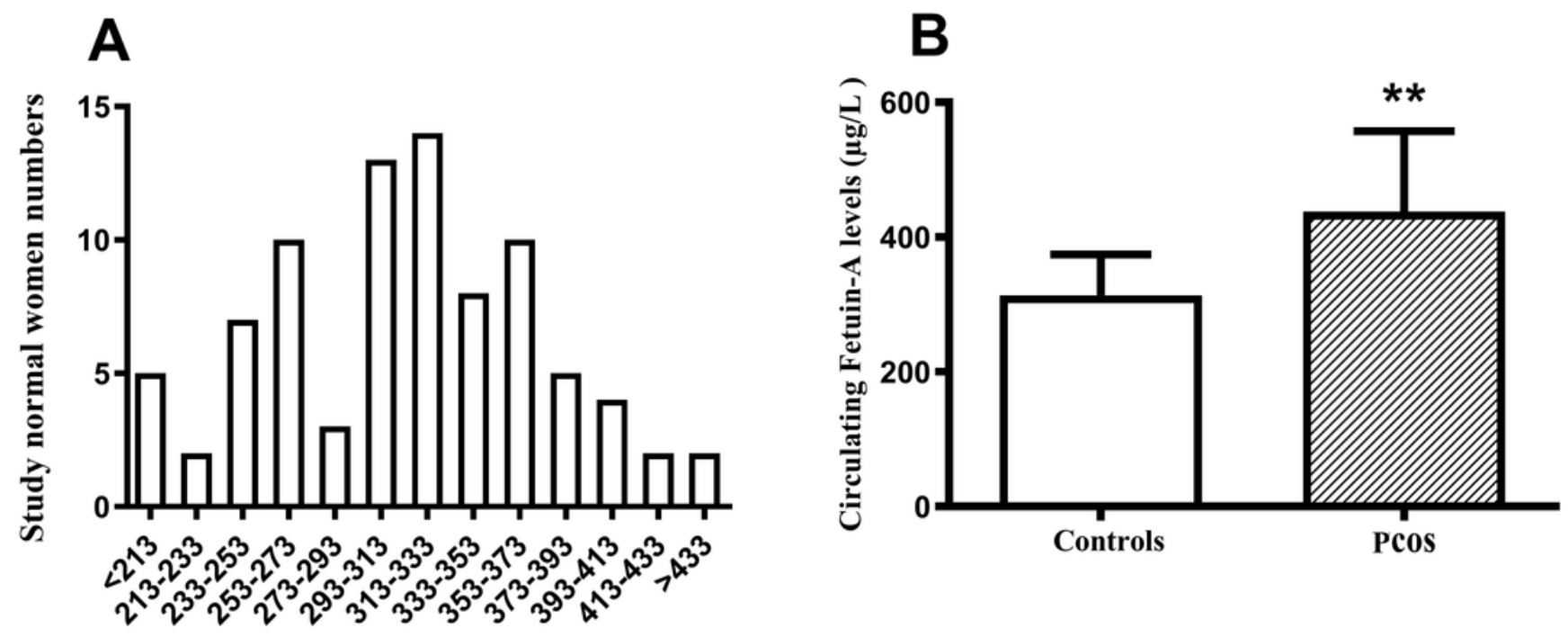

Circulating Fetuin-A levels $(\mu \mathrm{g} / \mathrm{L})$
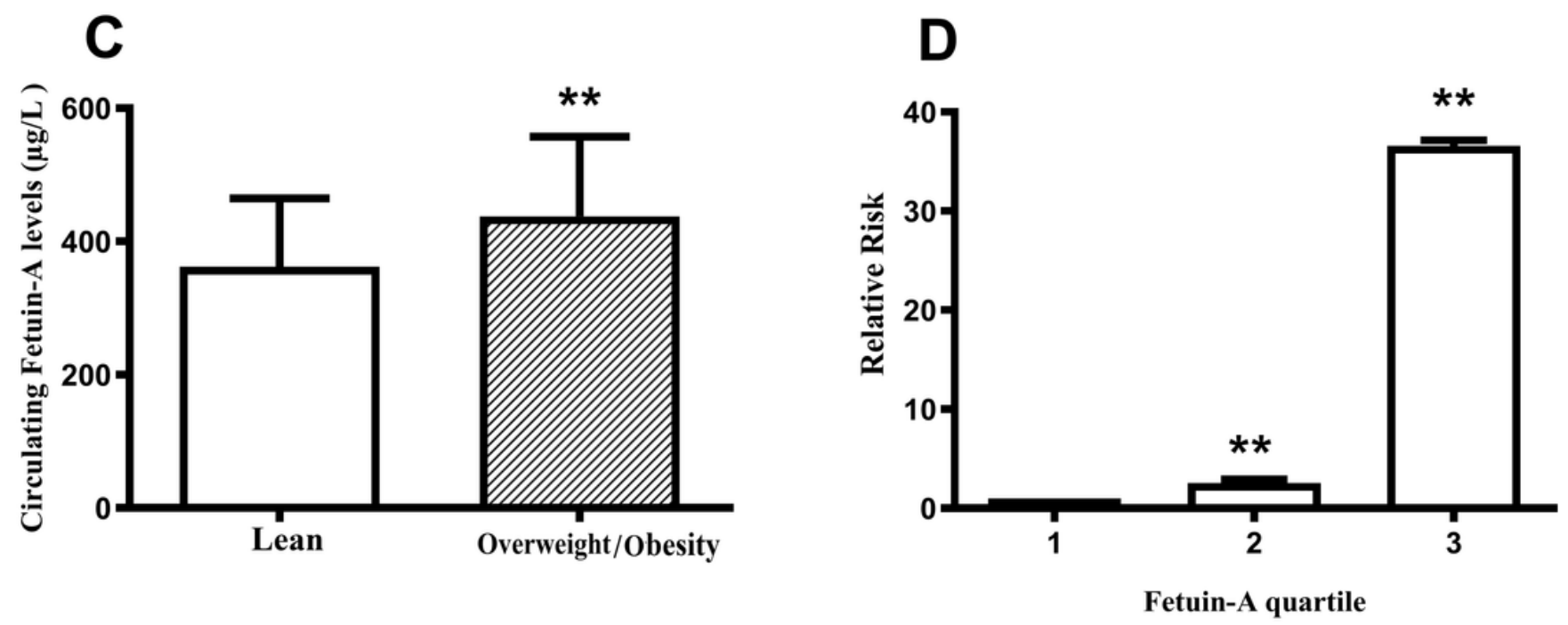

Figure 1

Serum Fetuin-A levels in the study population. (A) Distribution of circulating concentration of Fetuin-A in normal women. (B) Serum Fetuin-A levels in healthy and PCOS women. (C) Circulating Fetuin-A levels in lean and obese/overweight subjects. (D) The odds ratio of having MetS in different tertiles of circulating Fetuin-A. Data were expressed as means \pm SD. ${ }^{*} p<0.05,{ }^{\star *} p<0.01$ vs. Controls, lean or tertile 1 . 

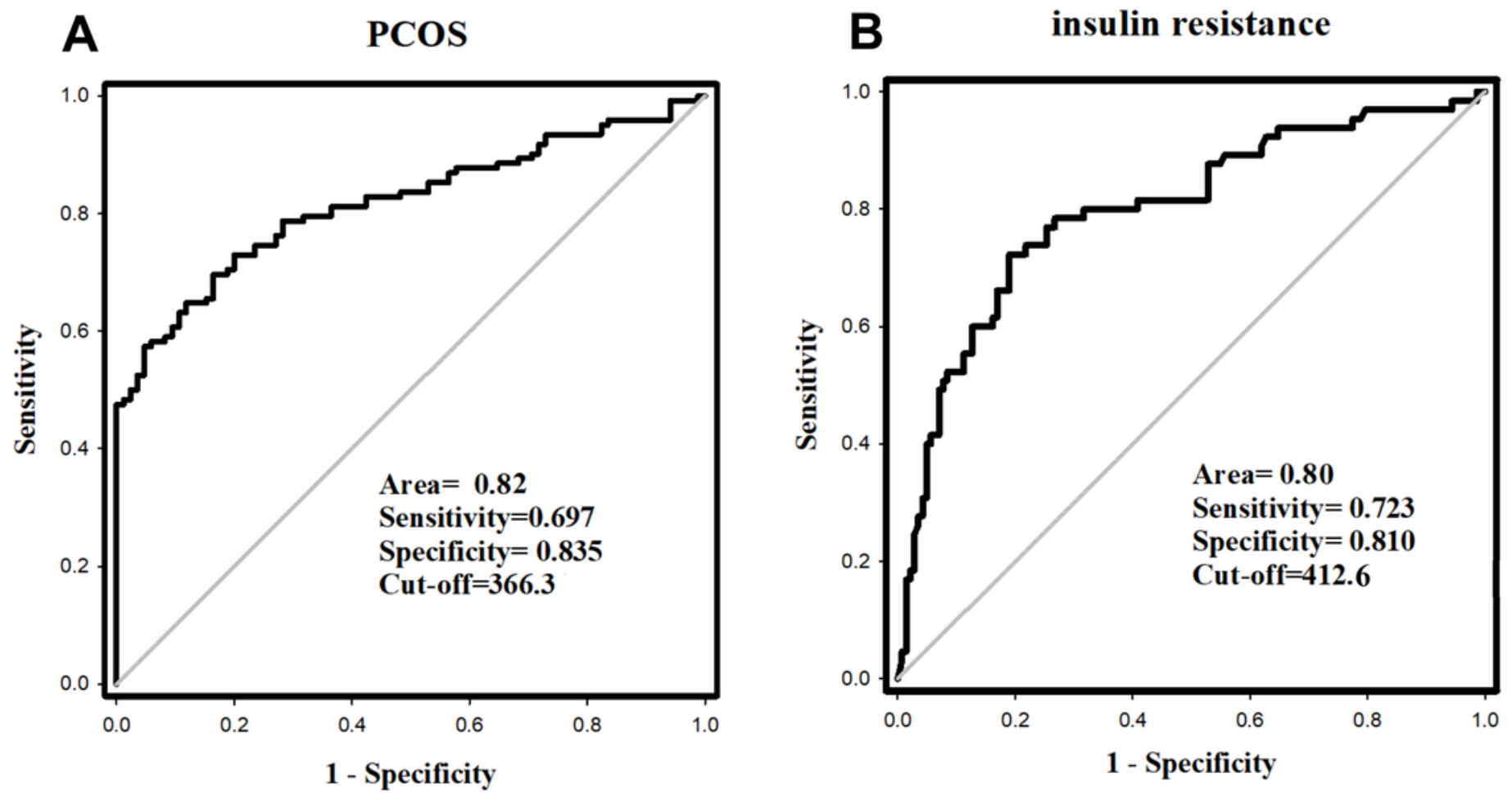

Figure 2

(A) ROC curve analysis of the prediction of PCOS. (B) ROC curve analysis of the prediction of insulin resistance. 